\title{
A Variability Based Classification for Microquasars
}

\section{Peggy Varniere}

AstroParticule \& Cosmologie (APC), UMR 7164, Université Paris Diderot, 10 rue Alice Domon et Lneonie Duquet, 75205 Paris Cedex 13, France

E-mail: varnieredapc.univ-paris7.fr

\section{J. Rodriguez}

CEA Saclay, DSM/IRFU/SAp, Laboratoire AIM F-91191 Gif sur Yvette France

E-mail: jerome.rodriguez@cea.fr

\section{Tagger}

LPCE, Orleans et AstroParticule \& Cosmologie (APC), UMR 7164, Université Paris Diderot, 10 rue Alice Domon et Lńeonie Duquet, 75205 Paris Cedex 13, France

E-mail: Michel.Taggerecnrs-orleans.fr

There has been a long string of efforts to classify observations of microquasars. The progress made in the understanding of the variability of these objects during the past few years makes it possible now, to reconsider this classification in terms of pysical processes. This will also have the interest of reuniting all microquasars in one classification.,Here we describe this classification based on the instabilities occurring in the disk and link them with the observed variability.

VII Microquasar Workshop: Microquasars and Beyond September 1-5 2008

Foca, Izmir, Turkey 


\section{Introduction}

Historically, black hole binaries were classified in five different states (quiescent, low, high, intermediate, very high), based on the shape of the energy spectrum (hereafter spectrum) and the flux level in the 1-10 keV band. This first attempt to classify the behavior of microquasars started with the observation of Cyg X1 and GX 339-4 and had for main parameter the flux, thought to be uniquely related to the mass accretion rate. As the flux (and therefore $\dot{M}$ ) increased the source was thought to go from quiescent to low/hard to intermediate to high/soft to very high. In 2004 and 2006 Remillard \& McClintock used the shape of the observed spectrum to define another set of three states and the transitions between them. This led to three states which they renamed in a more descriptive way (not based on the x-ray luminosity) as the Low-Hard state, the Steep Power Law state and the thermal state (High Soft state). XTE J1550-564 is a good example of this classification. It is interesting to note the strong link between their three classes and the temporal behavior of the source which could be used as an alternative description of the three states where the Thermal state does not have QPOs, the Low Hard State shows LFQPOs and the Steep Power Law has both LF and HF QPOs. This description was since refined using X-ray timing, especially the LFQPOs, and observations at other wavelength such as the presence of jet seen in radio. Homan \& Belloni, 2005, introduced a 4-state classification still in the continuity of those but with a stronger link to the observed LFQPOs. The Low-Hard State and the High-Soft state are in the direct continuity of the previous classification. The two other states in their classification are the Hard Intermediate which is the state in which a Low-Frequency QPO of C-type [3] is detected while the Soft Intermediate is characterized by either a B- or A-type LFQPO. This classification is coherent with an evolution in the HID from Low/Hard, Hard Intermediate, Soft Intermediate, High Soft Hard intermediate back to Quiescent/Low-Hard.

A different attempt was made in 2000 by Belloni et al.. They classified the behavior of the microquasar GRS $1915+105$ in 3 fundamental states A, B and C which combine into 12 reproducible classes $(\alpha, \beta, \ldots)$. Those three states, defined in a color-color diagram, are characterized by different contributions from the disk (thermal component of the X-ray) and its corona (non-thermal component at higher energy). This classification has proven useful, but is limited to that one particular object. The main difference between GRS 1915+105 and other microquasars is the occurrence of these cycles on short (up to a few tens of minutes) timescales. Looking at the temporal behavior of those three basic states, a one-to-one association seems to exist. Indeed, the A state of Belloni does not show any quasi-periodic oscillation while $\mathrm{C}$ always exhibits the Low-Frequency one. Finally, the B state seems to always be present in the case of HFQPO detection though no extensive study has been published yet. There exist no one-to-one connections between the previous state classification and this one, partially because GRS 1915+105 tends to stay at a much higher flux level than the other objects, making its state fits in the spectral classification much harder.

Both of these classifications have proven useful, but they are limited to certain objects. Here, we aim at a classification based on the physical processes possibly occuring in those objects. The first step in that direction was made with the Magnetic Flood Scenario (MFS, Tagger et al, 2004) which is a possible scenario for the $\beta$ class of GRS $1915+105$ based on the association of the Low- 
Frequency QPO (LFQPO) with the Accretion-Ejection Instability (AEI). The second step was to propose the Rossby-Wave Instability (RWI) as a possible mechanism at the origin of the HighFrequency QPO (HFQPO) in microquasars (Tagger \& Varniere, 2006). Here we will put together the different instabilities that could occur in a microquasar disk and compare the results with the existing classification.

\section{The Magnetic Flood Scenario: a three instabilities Scenario}

The Magnetic Flood Scenario (MFS, Tagger et al. 2004) was first introduced to explain the 30min cycle of GRS 1915+105 ( $\beta$ class of the Belloni et al. 2000 classification).

\subsection{The three instabilities at the origin of the three states}

The MFS starts with the identification of the Low Frequency Quasi-Periodic Oscillation with the Accretion-ejection Instability (AEI, Tagger \& Pellat 99). But, in order to explain this cycle we needed more than this association, which was only the first step.

In order to fully explain the observed state transitions, we needed to identify the three states from the physical conditions necessary for instabilities to develop. We were thus led to associate the state A with a turbulent disk, dominated by the Magneto-Rotational Instability (MRI).

Recently we proposed that the Rossby-Wave Instability (RWI) was at the origin of the HFQPO observed in microquasars (Tagger \& Varnière, 2006). Therefore a further interpretation of the B state of Belloni et al 2000, was called for, in which this state would be an RWI-dominated state with the disk close to its last stable orbit.

As a consequence we associated the three fundamental states of Belloni et al. 2000 with three distinct instabilities based on their properties and variabilities.

\subsection{Magnetic Flood Scenario}

The MFS explains the repetitive X-ray behavior as a limit cycle determined by the advection of poloidal magnetic flux to the inner disk and its destruction via magnetic reconnection with the magnetic flux trapped close to the source (which can lead to relativistic ejection).

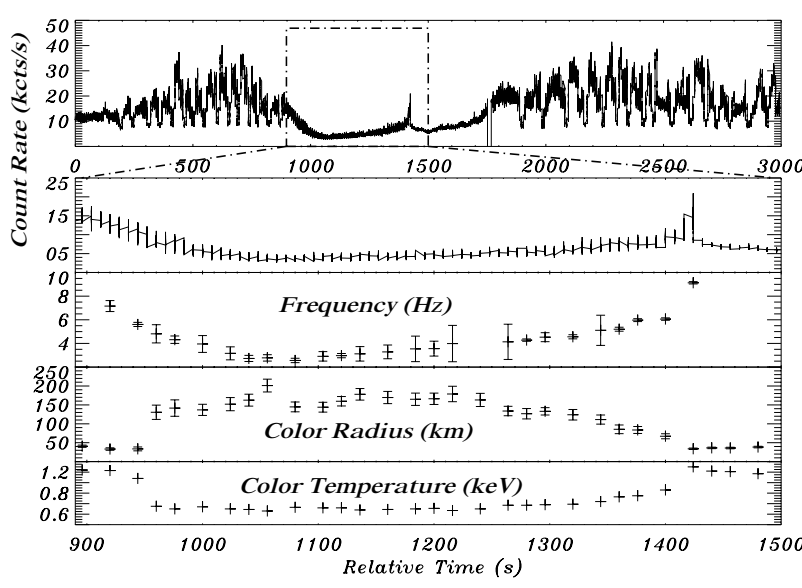

At the beginning of the cycle the source is in the high state. Accretion is caused by the Magneto-Rotational Instability which requires a low magnetic field. With accretion of the magnetic field, the $\beta$ parameter (magnetic pressure/gas pressure) decreases slowly, until it reaches $\beta \sim 1$. At that point the MRI stops and the AEI starts. the temperature decreases, further decreasing $\beta$ and the LFQPO appears. During the low state, the AEI is at the origin of the LFQPO and sends energy toward the corona by the means of Alfven waves [7]. 


\section{Three Instabilities but four states}

The RWI has the property of being a hydrodynamic instability (no magnetic field required) but to be stronger in the presence of a magnetic field. Also, in the case of a magnetized disk close to the equipartition, if the criteria for the RWI are fulfilled the criteria for the AEI will also be fulfilled. Therefore both instabilities can occur in the same disk, at two different locations, the RWI close to the last stable orbit and the AEI further away (depending on the parameter the frequency of the wave is a few tenths of the rotation at the inner edge of the disk).

We can use the parameters $\beta=8 \pi p / B^{2}$ and $\xi_{\text {int }}=r_{\text {int }} / r_{L S O}$ to decide which instability is dominant in the disk. With these two parameters, $\beta$ and $\xi$, we can map all the states in four cases which define four states of variabilities:

state $\beta>1, \xi_{\text {int }}>1$ : weakly magnetized disk far from its last stable orbit in which the MRI dominates. Observationally it is a turbulent disk with no QPOs (HF or LF). This state is similar to the High/Soft, Thermal or A state in the precedent classifications.

state $\beta>1, \xi_{\text {int }} \sim 1$ : weakly magnetized disk with its inner edge close to the last stable orbit. The RWI dominates. Observationally this should result in a warm disk with a HFQPO and no LFQPO.

state $\beta \sim 1, \xi_{\text {int }}>1$ : fully magnetized disk ( $\beta \sim 1$ ) with its inner edge far from the last stable orbit. This disk is dominated by the AEI. The characteristics of that state are a strong variable LFQPO, no HFQPO and a jet.

state $\beta \sim 1, \xi_{\text {int }} \sim 1$ : fully magnetized disk extending up to its last stable orbit. Both the AEI, and the magnetized version of the RWI are present in that disk, being at different position in the disk they can survive together but will interact and modify the characteristic of both. Observationally we have a not-too-variable LFQPO (inner radius is close to the last stable orbit) and a HFQPO.

From those four cases we can build four states to describe all the microquasar observations depending on the dominant instability (= the physical mechanism at the origin of the variability observed). The first three states were present in the Magnetic Flood Scenario. The forth state, with both the AEI and the RWI active, is a state with simultaneously a LFQPO and a HFQPO as it is so often the case in XTE J1550-564 during outbursts. This state was also observed recently in GRS $1915+105$ in the class $\theta$ by Belloni et al. 2006. So now GRS $1915+105$ exhibits the four variability states.

\section{Conclusion: A model Independent Look at the classification}

In this paper we propose a microquasar classification in four states which can be defined by the presence or the absence of both the HF and LF QPOs making the classification model independent. 


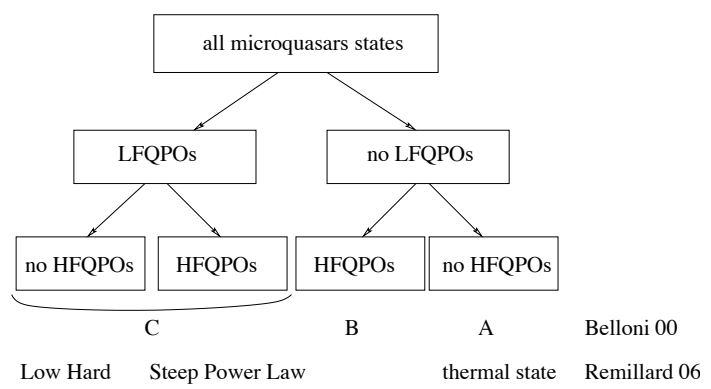

This classification is actually close to both the one by Belloni et al. and the one of Remillard et al. . The main difference with the classification based on GRS $1915+105$ is that we separate its $\mathrm{C}$ state in two states (AEI-dominated state and AEI-RWI-dominated state), depending on the high frequency variability. Since the original classification by Belloni in 2000, we now know that HFQPOs occur in the $\mathrm{C}$ state as defined by Belloni (see Belloni et al. 2006) so the AEI-MRIdominated state has been observed in GRS 1915+105. Concerning the classification based on XTE J1550-564 by Remillard et al., the main difference is the state with only HFQPO's which has not been observed yet.

The use of that classification has one major drawback. As it is based on physics and not on the observed quantities, it does not take into account the detection level. This becomes important for the state defined by the presence of the HFQPOs, which are known to be hard to detect. But this can be turned around and used as a HFQPO "predictor".

\section{References}

[1] Belloni et al., 2000, A\&A, 355, 271

[2] Belloni et al., 2006, MNRAS, 369, 305.

[3] Remillard et al., 2002, ApJ, 564, 962.

[4] R.A. Remillard \& J.E. McClintock, 2006, Ann. Rev. Astron. Astrophys. 44, 49.

[5] Tagger \& Pellat, 1999, A\&A, 349, 1003.

[6] M.Tagger et al., 2004, ApJ, 607, 410.

[7] Varniere \& Tagger, 2002, A\&A, 394, 329. 